\title{
Saturated long-chain fatty acid-producing bacteria contribute to enhanced colonic motility in rats
}

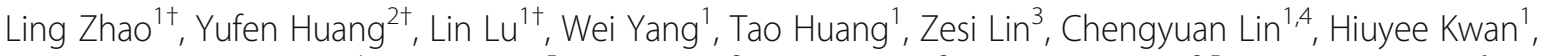
Hoi Leong Xavier Wong ${ }^{1}$, Yang Chen ${ }^{5}$, Silong Sun ${ }^{2}$, Xuefeng Xie ${ }^{2}$, Xiaodong Fang ${ }^{2,5}$, Huanming Yang ${ }^{6}$, Jian Wang ${ }^{6}$, Lixin Zhu ${ }^{7^{*}}$ and Zhaoxiang Bian ${ }^{1 *}$

\begin{abstract}
Background: The gut microbiota is closely associated with gastrointestinal (GI) motility disorder, but the mechanism(s) by which bacteria interact with and affect host Gl motility remains unclear. In this study, through using metabolomic and metagenomic analyses, an animal model of neonatal maternal separation (NMS) characterized by accelerated colonic motility and gut dysbiosis was used to investigate the mechanism underlying microbiota-driven motility dysfunction.

Results: An excess of intracolonic saturated long-chain fatty acids (SLCFAs) was associated with enhanced bowel motility in NMS rats. Heptadecanoic acid (C17:0) and stearic acid (C18:0), as the most abundant odd- and evennumbered carbon SLCFAs in the colon lumen, can promote rat colonic muscle contraction and increase stool frequency. Increase of SLCFAs was positively correlated with elevated abundances of Prevotella, Lactobacillus, and Alistipes. Functional annotation found that the level of bacterial LCFA biosynthesis was highly enriched in NMS group. Essential synthetic genes Fabs were largely identified from the genera Prevotella, Lactobacillus, and Alistipes. Pseudo germ-free (GF) rats receiving fecal microbiota from NMS donors exhibited increased defecation frequency and upregulated bacterial production of intracolonic SLCFAs. Modulation of gut dysbiosis by neomycin effectively attenuated GI motility and reduced bacterial SLCFA generation in the colon lumen of NMS rats.

Conclusions: These findings reveal a previously unknown relationship between gut bacteria, intracolonic SLCFAs, and host GI motility, suggesting the importance of SLCFA-producing bacteria in Gl motility disorders. Further exploration of this relationship could lead to a precise medication targeting the gut microbiota for treating Gl motility disorders.
\end{abstract}

Keywords: Gastrointestinal motility disorder, Gut microbiota, Neonatal maternal separation, Saturated long-chain fatty acids

\section{Background}

Disordered gastrointestinal (GI) motility is one major symptom frequently presented in patients suffering with functional GI disorders (FGIDs) and other bowel

\footnotetext{
* Correspondence: lixinzhu@buffalo.edu; bianzxiang@gmail.com

${ }^{\dagger}$ Ling Zhao, Yufen Huang and Lin Lu contributed equally to this work.

${ }^{7}$ Digestive Diseases and Nutrition Center, Department of Pediatrics, The State University of New York at Buffalo, 3435 Main Street, 422BRB, Buffalo, NY 14214, USA

${ }^{1}$ Chinese Medicine Clinical Study Center, Jockey Club School of Chinese Medicine, Hong Kong Baptist University, Kowloon Tong, Hong Kong SAR, China

Full list of author information is available at the end of the article
}

diseases [1, 2]. Chronic or recurrent episodes of dysregulated GI motility severely impact patients' quality of life [3]. The underlying mechanisms of disordered GI motility are multifaceted. Previous studies have revealed contributions of immune activation, ionic channels and neurohumoral dysregulation to GI motor dysfunction [4-6]. However, current etiological understanding of disordered GI motility is incomplete, which limits the development of personalized and precisely effective medicine for GI motility disorders.

(c) The Author(s). 2018 Open Access This article is distributed under the terms of the Creative Commons Attribution 4.0 International License (http://creativecommons.org/licenses/by/4.0/), which permits unrestricted use, distribution, and reproduction in any medium, provided you give appropriate credit to the original author(s) and the source, provide a link to the Creative Commons license, and indicate if changes were made. The Creative Commons Public Domain Dedication waiver (http://creativecommons.org/publicdomain/zero/1.0/) applies to the data made available in this article, unless otherwise stated. 
The gut microbiota is thought to be an important factor associated with disordered GI motility [7]. For example, patients with irritable bowel syndrome (IBS), especially for diarrhea-predominant IBS (IBS-D), showed a lower diversity and a higher instability of gut microbial community $[8,9]$. Altered composition of the gut microbiota was also found in children and adults with chronic functional constipation [10-12]. Regulation of the gut microbiota by probiotics can improve bowel movement frequency in up to $70 \%$ of functionally constipated patients [13]. Transplantation of fecal microbiota from IBS-D suffers significantly accelerated colonic transit in germ-free (GF) mice [14]. GF mice colonized with fecal microbiota from patients with slow transit constipation exhibit lower fecal frequency, delayed GI transit time, and weaker spontaneous contractions of colonic smooth muscle [15]. These results point directly to a conclusion that the gut microbiota serves a pivotal role in GI motility disorder. But the mechanism by which microbiota interact with and affect host GI motility remains unclear.

It has been proposed that the effect of the gut microbiota on host GI motility partly derives from release of bacterial end-products of fermentation or molecules derived from host and gut microbiota co-metabolism [16, 17]. One study found that microbial products, such as lipopolysaccharides (LPS), can regulate GI motility through activating toll-like receptor 4 (TLR4) signaling that increases survival of nitrergic neurons [18]. Short-chain fatty acids and lactic acids, produced from bacterial fermentation of dietary fiber and resistant starch, can regulate intestinal motor action, probably through regulating peptide YY secretion and cholinergic neurons [19]. In another study, GF mice displayed accelerated GI motility after colonization with spore-forming microbes, the metabolites of which could stimulate enteric serotonin release [20]. Therefore, gut microbiota-derived substances appear to be the link between gut microbiota and host GI motility.

A rodent model of neonatal maternal separation (NMS) is a well-established model characterized by long-term colonic dysfunction [21]. Our previous study found that colon tissue isolated from adult NMS rats showed a higher level of muscle amplitude [22]. Such enhanced colonic motility is involved in upregulation of L-type calcium channels in colonic smooth muscle cells [22] and increased serotonin production from colonic enterochromaffin cells (ECs) [23]. Meanwhile, gut microbial disturbance is also reported in both mice and rats subjected to NMS [24, 25], but the association of changed gut microbiota with colonic dysmotility in an NMS model is yet to be investigated.

In this study with an NMS rat model, metabolic profiling of feces and luminal contents of the GI tract of experimental animals was preformed to identify featured microbiota-derived metabolites. A group of saturated long-chain fatty acids (SLCFAs) was remarkably increased in the colon lumen and feces of NMS rats. After confirming their stimulatory effects on colon motility, function-based metagenomic analysis was done and found hyperactive synthesis of SLCFAs by the gut microbiota of NMS rats. The relationship between SLCFA-producing bacteria and host colon motility were further clarified by transplanting fecal microbiota from NMS donors into pseudo germ-free (GF) rats and modulating gut microbiota in NMS rats by neomycin intervention. Based on these experiments, we aimed to clarify the linkage between gut microbiota and host colonic motility phenotype and to determine which bacteria are the primary agents manipulating host GI colonic dysmotility.

\section{Results}

Excess intracolonic SLCFAs are associated with GI motility in NMS model

The experimental procedure for establishing NMS model was portrayed in Fig. 1a. Compared with controls, increased stool frequency and shortened gut transit time were observed in adult rats subjected to NMS, suggesting enhanced GI motility (Fig. 2a, b). There was no difference in body weight between the groups (Additional file 1: Figure S1C), but fecal samples of NMS rats showed higher water contents and lower $\mathrm{pH}$ (Additional file 1: Figure $\mathrm{S} 1 \mathrm{~A}$ and $\mathrm{B})$, which can be interpreted as abnormal fecal characteristics. The fecal volatile organic compounds (VOCs) were profiled by non-targeted metabolomic analysis by gas chromatography coupled with mass spectrum (GC-MS). Partial least squares-discriminant analysis (PLS-DA) showed pronounced differences in the profiles of fecal VOCs between the two groups (Fig. 2c). A total of 21 changed metabolites in NMS rats was identified referred by the variable importance in the projection (VIP) $>1$ and $p<0.05$ (Additional file 1: Table S1). Of these metabolites found, 11 were acidic substances closely associated with gut microbial fermentation and energy metabolism. Particularly, the six saturated fatty acids (SFAs) of the two groups differed dramatically, with increase of heptadecanoic acid (C17:0), tetradecanoic acid (C14:0), pentadecanoic acid (C15:0), and acetic acid (C2:0) while decrease of butyric acid (C4:0) and valeric acid (C5:0) in the NMS rats (Fig. 2d). These alterations suggest disturbance of the luminal SFA composition in NMS rats. Further, targeted SFA analysis found that the level of total SFAs was remarkably increased in the colon lumen of NMS rats (Fig. 2e). The cumulative levels of saturated short-chain fatty acids (from $\mathrm{C} 2: 0$ to $\mathrm{C5}: 0$ ) and medium-chain fatty acids (from C6:0 to C12:0) were unchanged (Fig. 2f), but the level of total and individual SLCFAs (from C14:0 to C18:0) showed significant over-representation (Fig. 2f, g). These results suggest that 


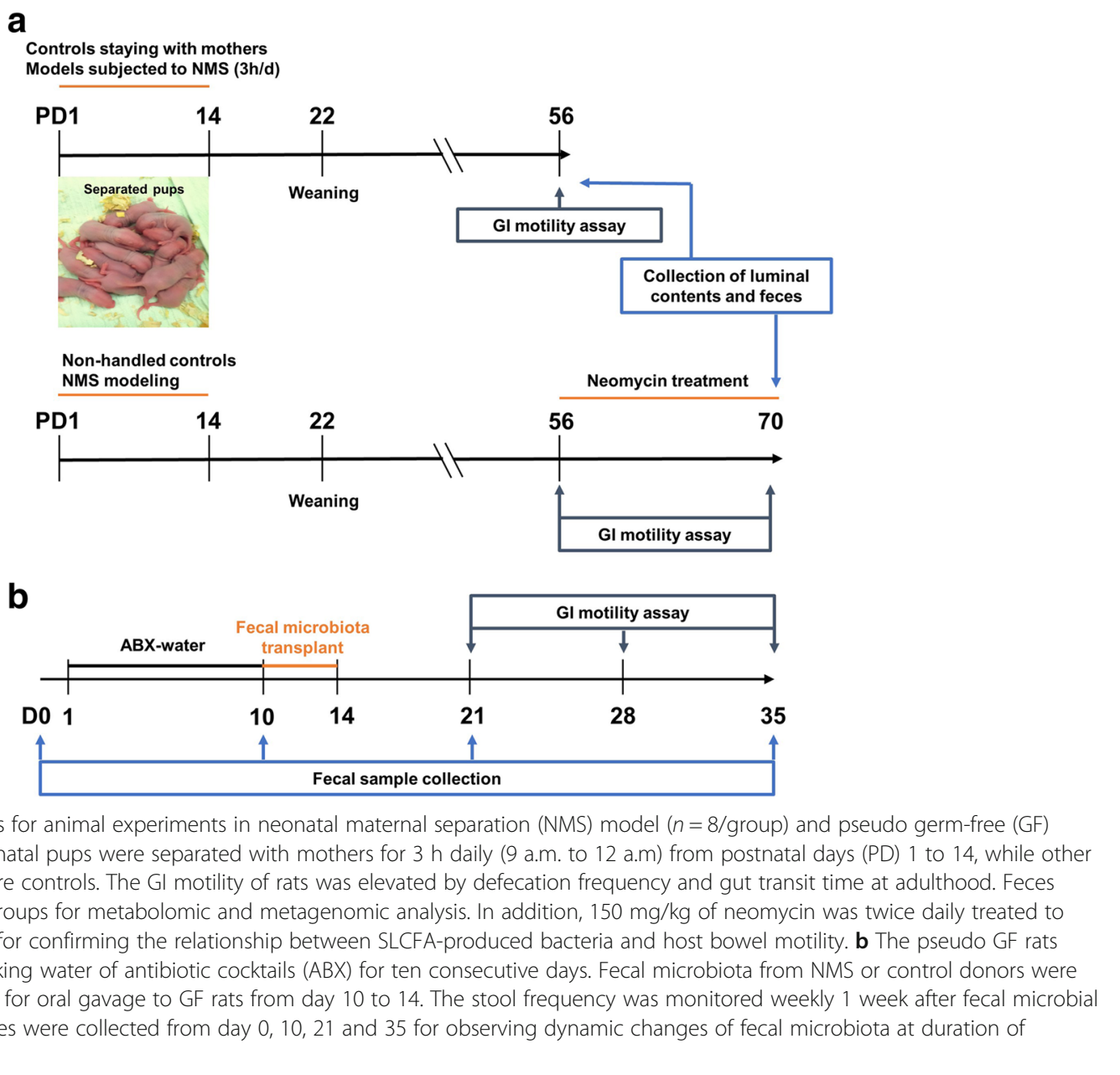

Fig. 1 The detail procedures for animal experiments in neonatal maternal separation (NMS) model ( $n=8 /$ group) and pseudo germ-free (GF) model ( $n=6$ /group). a Neonatal pups were separated with mothers for 3 h daily (9 a.m. to 12 a.m) from postnatal days (PD) 1 to 14 , while other pups staying with mother are controls. The Gl motility of rats was elevated by defecation frequency and gut transit time at adulthood. Feces were collected from both groups for metabolomic and metagenomic analysis. In addition, $150 \mathrm{mg} / \mathrm{kg}$ of neomycin was twice daily treated to NMS rats from PD 56 to 70 for confirming the relationship between SLCFA-produced bacteria and host bowel motility. b The pseudo GF rats model was induced by intaking water of antibiotic cocktails (ABX) for ten consecutive days. Fecal microbiota from NMS or control donors were prepared as PBS suspension for oral gavage to GF rats from day 10 to 14 . The stool frequency was monitored weekly 1 week after fecal microbial transplantation. Fecal samples were collected from day 0, 10, 21 and 35 for observing dynamic changes of fecal microbiota at duration of the experiment

an excess of intracolonic SLCFAs is associated with enhanced GI motility in NMS rats.

\section{SLCFAs stimulate rat colonic contraction and defecation} Lipid perfusion has been shown to enhance colon motility in normal subjects and patients with IBS-D [26]; however, the effects of SLCFAs on colonic motility have not been reported. To determine these effects, we studied C17:0 and C18:0, the most abundant even- and odd-numbered carbon SLCFAs in the colon lumen of NMS rats, using an organ bath system as described in our previous study [27]. Compared with baseline (fatty acid-free BSA), C17:0 (50 and $100 \mu \mathrm{M}$ ) and C18:0 (30, 50 , and $100 \mu \mathrm{M})$ dose-dependently enhanced contraction amplitudes of colonic circular muscles (Fig. 3a, b). Acetylcholine treatment was used as the positive control (Additional file 1: Figure S2A). C2:0, another saturated FA found to be increased level in fecal VOCs of NMS rats, was tested as a negative control (Additional file 1: Figure S2B). It is well known that GPR40 and GPR120, expressed in the endocrine cells of the colon, can be activated by free LCFAs [28-30]. To test which free long-chain fatty acid receptors are involved in such SLCFA-induced muscle contraction, isolated colonic segments were separately treated with different doses of DC260126 (GPR40 antagonist) and AH7614 (GPR120 antagonist) prior to introduction of C18:0. We found that DC260126 in doses of 5 and $10 \mu \mathrm{M}$ can effectively suppress C18-induced colonic contraction, but AH7614 showed no effects (Fig. 3c and Additional file 1: Figure S2B). Furthermore, this effect of SLCFAs on colonic motility was confirmed in normal rats in vivo through orally administrating SLCFAs at dosages of $1,2.5$, and $5 \mathrm{mg} /$ $\mathrm{kg}$. Both C17:0 (5 mg/kg) and C18:0 (2.5 and $5 \mathrm{mg} / \mathrm{kg})$ notably increased rat defecation frequency (Fig. 3d, e). The cumulative amount of intracolonic SLCFAs was significantly increased in rats given $5 \mathrm{mg} / \mathrm{kg}$ of $\mathrm{C} 17$ or $\mathrm{C} 18$ (Fig. 3f), and this amount is close to the total SLCFA level in the colonic contents from NMS rats. These findings revealed that SLCFAs can accelerate rat colonic motility, and such action is possibly involved in GPR40 signaling. 

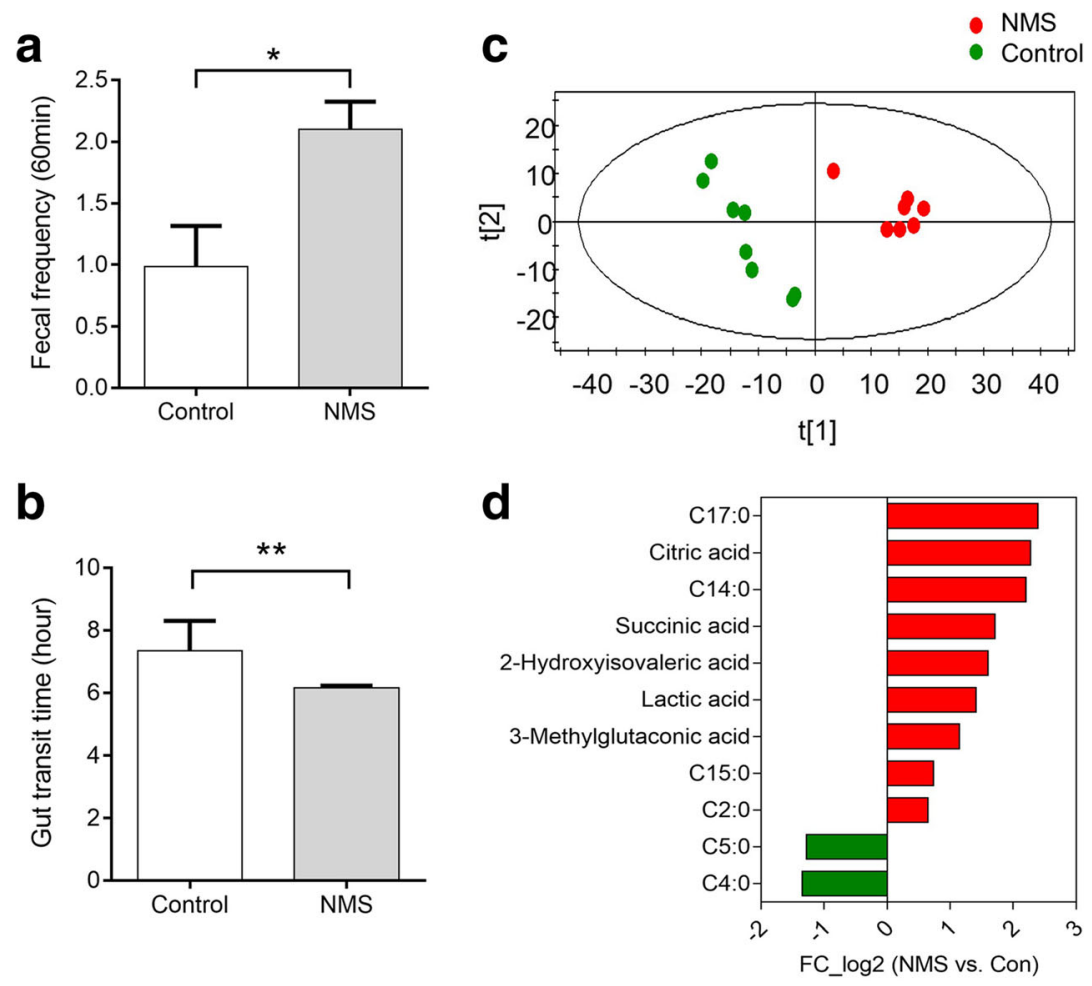

e

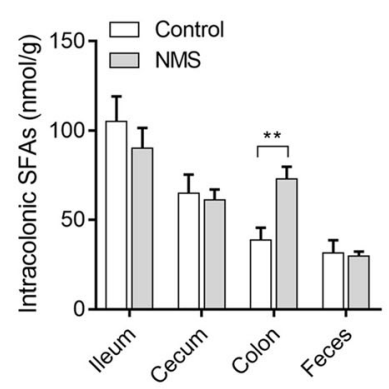

$\mathbf{f}$

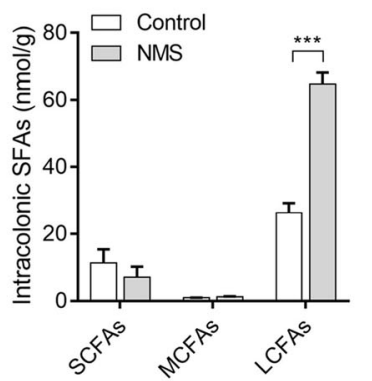

g

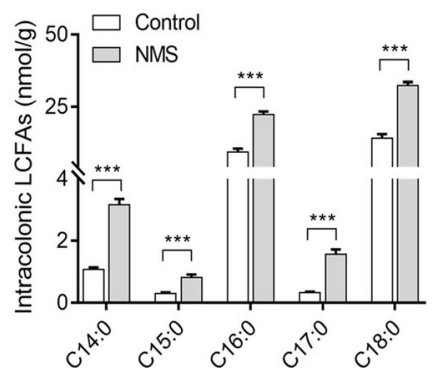

Fig. 2 NMS rats are characterized by enhanced gut motility and upregulated intracolonic saturated long-chain fatty acids (SLCFAs) relative to controls ( $n=8 /$ group). a Accumulation of fecal pellet output within $60 \mathrm{~min}$. b The gut transit time measured by oral administration of carmine red marker. $\mathbf{c}$ The scatter plot of fecal volatile organic compounds (VOCs) based on partial least squares discriminant analysis (PLS-DA). $X$ and $Y$ axes (t1 and t2) indicate the first two discriminating vectors, which respectively explain 33.5 and $16.6 \%$ of variation in the dataset. $\mathbf{d}$ Alteration of acidic substances derived from microbial metabolism in NMS rats. e The level of total saturated fatty acids (SFAs) per gram of luminal contents collected from ileum, cecum, proximal colon and feces. $\mathbf{f}$ The total levels of saturated short-chain, medium-chain, and long-chain fatty acid per gram of colonic contents in rats. $\mathbf{g}$ The level of individual SLCFAs per gram of colonic contents in rats. C2:0, acetic acid; C4:0, butyric acid; C5:0, valeric acid; C14:0, tetradecanoic acid; C15:0, pentadecanoic acid; C16:0, hexadecanoic acid; C17:0, heptadecanoic acid; C18:0, octadecanoic acid. Bar charts are plotted using mean \pm SEM value, and statistical significance between both groups is defined as ${ }^{*} p<0.05 ;{ }^{* *} p<0.01$; ${ }^{* * *} p<0.005$

\section{Changed fecal microbiome is associated with SLCFA generation in NMS rats}

Generally, intraluminal SLCFAs are mainly derived from diets or synthesized by host and gut microbiota. A previous study screened six genes (Fasn, Scd1, Srebp1, CD36, Fabp2, and Cav1) related to lipogenesis and lipolysis based on RT-PCR array and found no difference in their levels between adult NMS and control rats [31]. These evidences indicate that NMS rats have normal levels of host fatty acid synthesis, digestion, and absorption. Another piece of evidence has revealed that odd-numbered carbon SLCFA C15:0 and C17:0 can be produced by bacteria only [32]. Hence, we hypothesized that increase of SLCFAs is associated with the gut microbiota.

Gut dysbiosis in NMS rodents has been previously reported based on 16s ribosomal RNA sequencing [24, 25]. However, such approach cannot be used to obtain information related to metabolic function. To investigate whether gut dysbiosis results in abnormal SLCFAs, and 

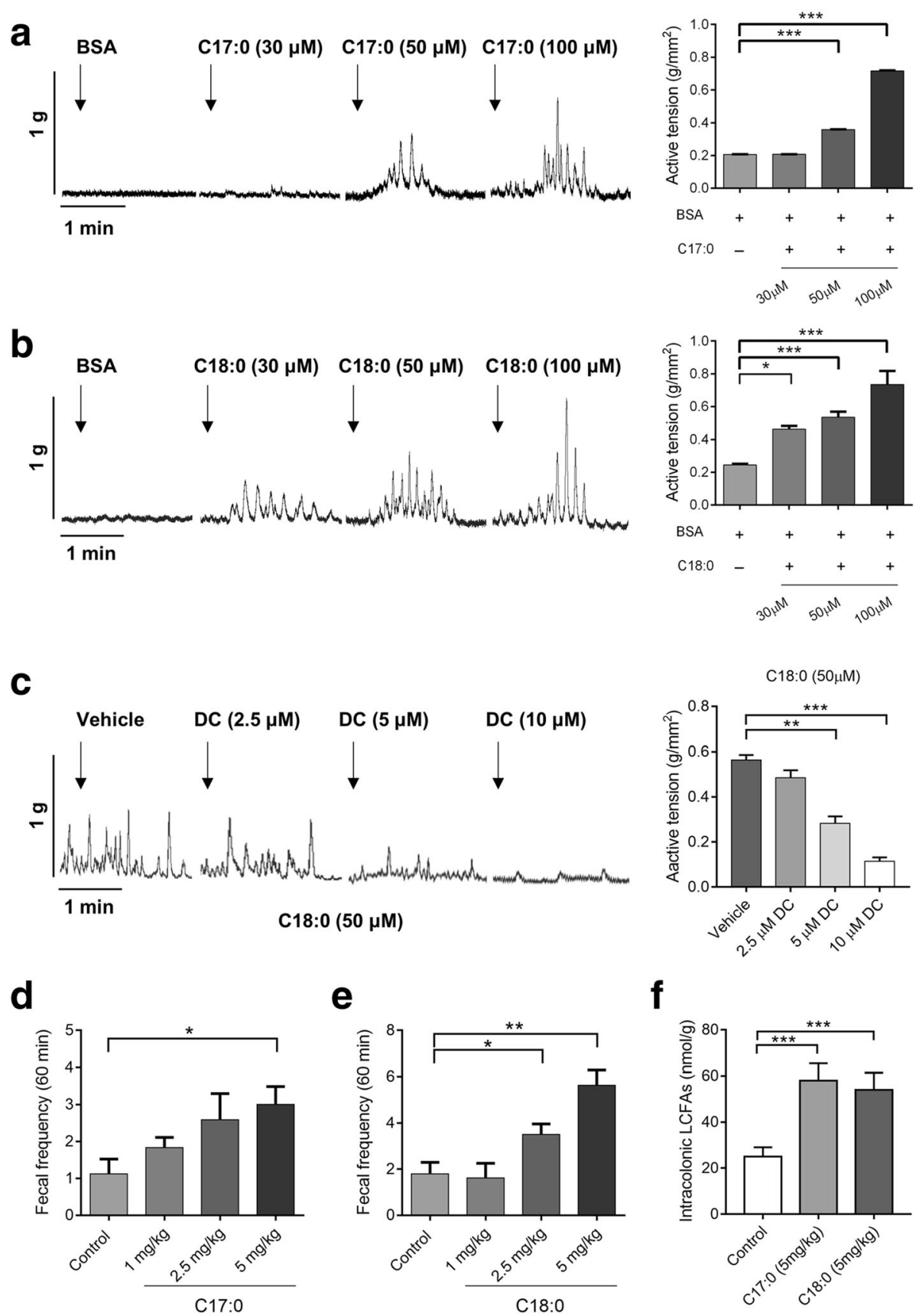

Fig. 3 C17:0 and C18:0 dose-dependently stimulate colonic motility ex vivo and in vivo, and such SLCFA-induced muscle contraction can be attenuated by TLR4 inhibitor C34. a, b The effects of C17:0 and C18:0 on the contraction of circular muscles of isolated colon segments through an organ bath system. The amplitude of contractions was expressed as force/area $\left(\mathrm{g} / \mathrm{mm}^{2}\right)$. c The effects of selective GPR40 antagonist DC260126 (DC) on C18:0-induced colonic muscle contraction. $\mathbf{d}$, e The effects of C17:0 and C18:0 on the accumulation of fecal pellet output within 60 min in normal rats ( $n=8 /$ group). $\mathbf{f}$ The accumulated amounts of SLCFAs per gram of colonic contents in SLCFA-treated and vehicle rats. Statistical differences among individual groups were evaluated using One-way ANOVA, and significance is defined as ${ }^{*} p<0.05 ;{ }^{* *} p<0.01 ;{ }^{* * *} p<0.005$

what the key manipulators are, we performed metagenomic sequencing analysis of fecal samples collected on postnatal day (PD) 56 from NMS and control rats $(n=$ $8 /$ group). In total, $83.01 \%$ of the high-quality sequencing reads (6.6 GB per sample on average) were used to generate 2.7 million contigs without ambiguous bases (minimum length of $500 \mathrm{bp}$ ), which allowed on average $74.75 \%$ of the reads in each sample to be mapped (Additional file 2: Table S2). Metagenomic results showed that microbial richness and $\alpha$-diversity was reduced in 
NMS rats at gene level (Additional file 1: Figure S3A). Microbial $\beta$-diversity was distinct between both groups based on either principal coordinate analysis (PCoA) analysis (Fig. 4a) or Bray-Curtis dissimilarity (Additional file 1: Figure S3B). Taxonomy of the microbes was profiled at phylum, genus, and species levels (Additional file 2: Tables S3-S5). At the phylum level, Bacteroidetes, Firmicutes, Proteobacteria, Deferribacteres, and Actinobacteria dominated the fecal microbial communities of both groups, but without statistical differences (Additional file 1: Figure S3C). Taxonomic profiles of both groups were compared at genus level (Additional file 1: Figure S3D) and revealed that relative abundances of Bacteroides, Blautia, and Parabacteroides were slightly decreased, while abundances of Prevotella, Lactobacillus, Alistipes, and Ruminiclostridum were significantly increased in fecal microbiota of NMS rats (Fig. 4b). At species level, 168 species were significantly altered, and a majority of them (90 species) were found significantly correlated with at least one of the fecal SLCFAs (Additional file 2: Table S6). More specifically, multiple species from the genera Prevotella, Lactobacillus, and Alistipes were positively correlated with fecal C17:0 or/and C14:0 (Spearman's correlation, adjusted $p$ $<0.05$; Fig. 4c and Additional file 2: Table S6). Moreover, pathway analysis of gene and genome (KEGG) orthologs (KO) found that the levels of bacterial fatty acid biosynthesis and elongation were highly enriched in the NMS model group, whereas fatty acid degradation level appeared no difference in NMS rats in comparison to controls (Fig. 4d). Ten genes mapped in fatty acid synthesis were identified in both groups. Of these, five genes, encoding to synthetases and elongases (FabD, FabF, FabG, FabZ, and FabI) responsible for bacterial LCFA synthesis and elongation [33, 34], showed elevated relative abundances in the NMS group (Fig. 4e). Moreover, abundances of genes TesA, YciA, and FadL, encoding to thioesterase and long-chain fatty acid transporter that takes charge of termination of LCFA elongation and LCFA transport across bacterial cytoplasmic membranes $[35,36]$, were also elevated in the NMS group (Fig. 4e). Notably, the abundant elongating Fab genes that are indispensable for LCFA generation were expressed in the genera Prevotella, Lactobacillus, and Alistipes (Additional file 2: Table S7), suggesting that they participate SLCFA formation. In addition, five Fad genes encoding to oxidase enzymes were mapped in fatty acid $\beta$-oxidation, one important pathway for LCFA degradation. The level of FadA was significantly reduced in the NMS group while other genes showed no difference (Fig. 4f). Taken together, metagenomic results suggest hyperactive bacterial SLCFA synthesis in NMS rats, with contributions from the genera Prevotella, Lactobacillus, and Alistipes.

\section{Fecal microbiota from NMS donors enhances stool} frequency and intracolonic SLCFAs in pseudo GF rats To investigate whether gut microbiota of NMS rats promotes SLCFA production in the colon lumen and enhances bowel motility, fecal microbiota prepared from NMS or NH donors were transplanted to pseudo GF rats ( $n=6 /$ group). The experimental process is showed in Fig. 1b. The pseudo GF model was induced by providing rats with water spiked with antibiotic cocktail (ABX), according to a published method [37]. During GF model establishment, rats from different cages consumed similar volumes of $A B X$ water (Additional file 1: Figure S4A), indicating there would be no difference in bactericidal action for GF modeling. Fecal microbiota extracted from donors were prepared as PBS suspensions, and orally administrated to pseudo GF rats for 5 consecutive days. One week after fecal microbial transplant (FMT), accumulation of fecal output within $60 \mathrm{~min}$ was found to be significantly increased in GF rats colonized with microbiota of NMS donors (NMS FMT) relative to controls that has been colonized with microbiota of control rats (Control FMT); the change was maintained over the following 2 weeks (Fig. 5a). In addition, the baseline of defecation number was higher in GF recipients than conventional rats, which possibly relates to watery or shapeless stools presumably caused by continuous infusion of ABX water. A similar fecal phenotype has been reported in other antibiotic-induced GF studies [38, 39].

Microbial DNA extracts from fecal samples of GF rats on day 0 (baseline), day 10 (after ABX treatment), day 21 ( 1 week after transplant), and day 35 ( 3 weeks after transplant) were subjected to $16 \mathrm{~s}$ ribosomal RNA sequencing analysis to determine the dynamic change of fecal enterotypes (Additional file 2: Table S8). Compared with the baseline, dramatic loss of microbial richness and DNA integrity observed in ABX-treated rats indicates that the antibiotic-induced pseudo GF model was well-developed (Additional file 1: Figure S4A-C). The ecological community of feces collected from colonized GF rats was then compared with that of the donors from diversity and taxonomic perspectives. PCoA analysis showed a certain similarity of bacterial $\beta$-diversities between recipients and donors (Additional file 1: Figure S4D). Specifically, changes of 49 genera from NMS donors, accounting for $50 \%$ of identified bacteria, also appeared in GF rats with NMS FMT (Additional file 2: Table S9).

Although the bacterial $\alpha$-diversity from both groups of recipients was similar (Additional file 1: Figure S4B), both $\beta$-diversity and bacterial composition in GF rat with NMS FMT were certainly distinct from those that had received control FMT (Additional file 1: Figure S4D and E). Particularly, relative abundances of Prevotella, Lactobacillus, and Alistipes were significantly 

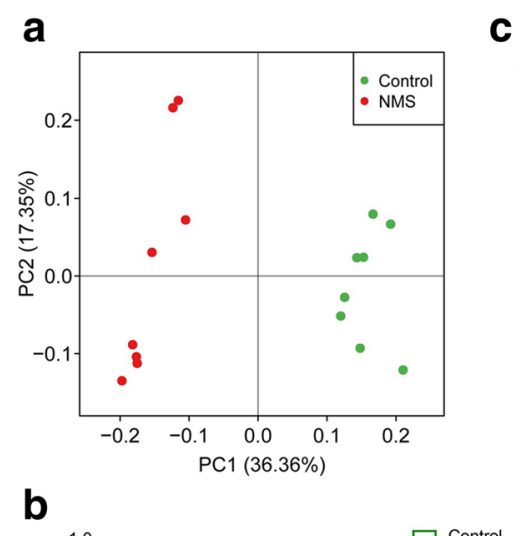

b

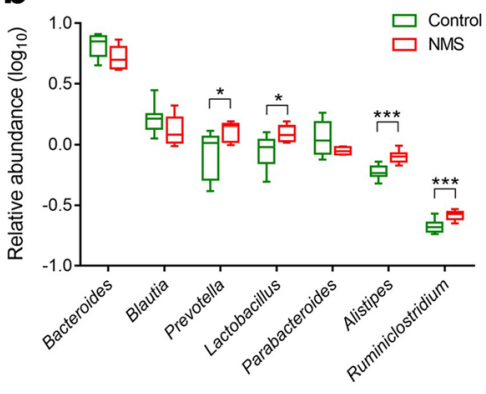

d

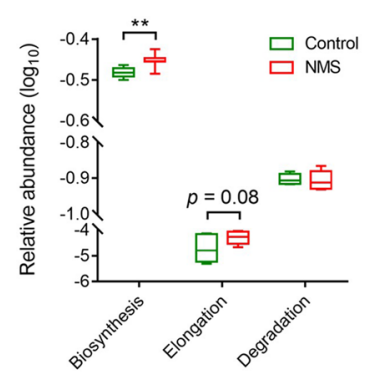

e

Bacterial bioysnthesis
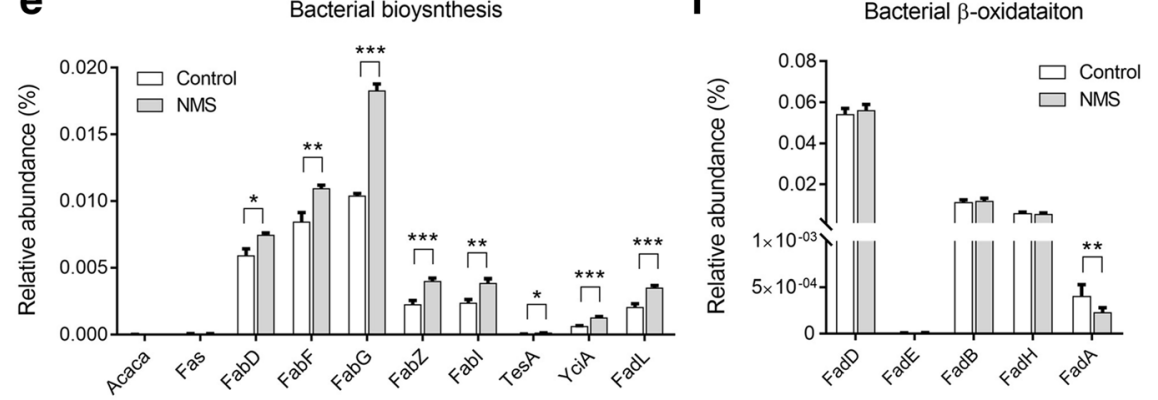

Fig. 4 Alteration of fecal microbiome is closely associated with SLCFA generation in NMS rats ( $n=8 /$ group). a Principal coordinate analysis (PCoA) of microbial beta-diversity between both groups. The first two principal coordinates (PCs) respectively explain $36.36 \%$ and $17.35 \%$ of variation in the dataset. $\mathbf{b}$ The relative abundances of dominant genera in fecal microbiota. $\mathbf{c}$ The spearman's correlation between changed fecal species and fecal C15:0, C17:0, C14:0. Only species correlated with at least one SLCFA with adjusted $p<0.05$ are shown in the heatmap. $\mathbf{d}$ The relative abundances of fatty acid biosynthesis, elongation and degradation based on $\mathrm{KO}$ module analysis. e The relative abundances of genes encoding enzymes for SLCFA synthesis. $\mathbf{f}$ The relative abundances of genes encoding enzymes for $\beta$-oxidation. Differential abundance of taxa and KOs were evaluated by two-tailed Wilcoxon rank-sum test, and significance is defined as ${ }^{*} p<0.05$; ${ }^{* *} p<0.01$; ${ }^{* *} p<0.005$. Abbreviation: Acaca, acetyl-CoA carboxylase; Fas, fatty acid synthase (bacteria type); FabD, Malonyl CoA-acyl carrier protein; FabF, 3-oxoacyl-[acyl-carrier-protein] synthase II; FabG, 3-oxoacyl-[acyl-carrier protein] reductase; FabZ, 3-hydroxyacyl-[acyl-carrier- protein] dehydratase; Fabl, enoyl-[acyl-carrier protein] reductase I; TesA, acyl-CoA thioesterase I; YciA, acyl-CoA thioesterase; FadL, long-chain fatty acid transport protein; FadD, long-chain acyl-CoA synthetase; FadE, acylCoA dehydrogenase; FadB, 3-hydroxybutyryl-CoA dehydrogenase; FadH, 2,4-dienoyl-CoA reductase; FadA, acetyl-CoA acyltransferase 

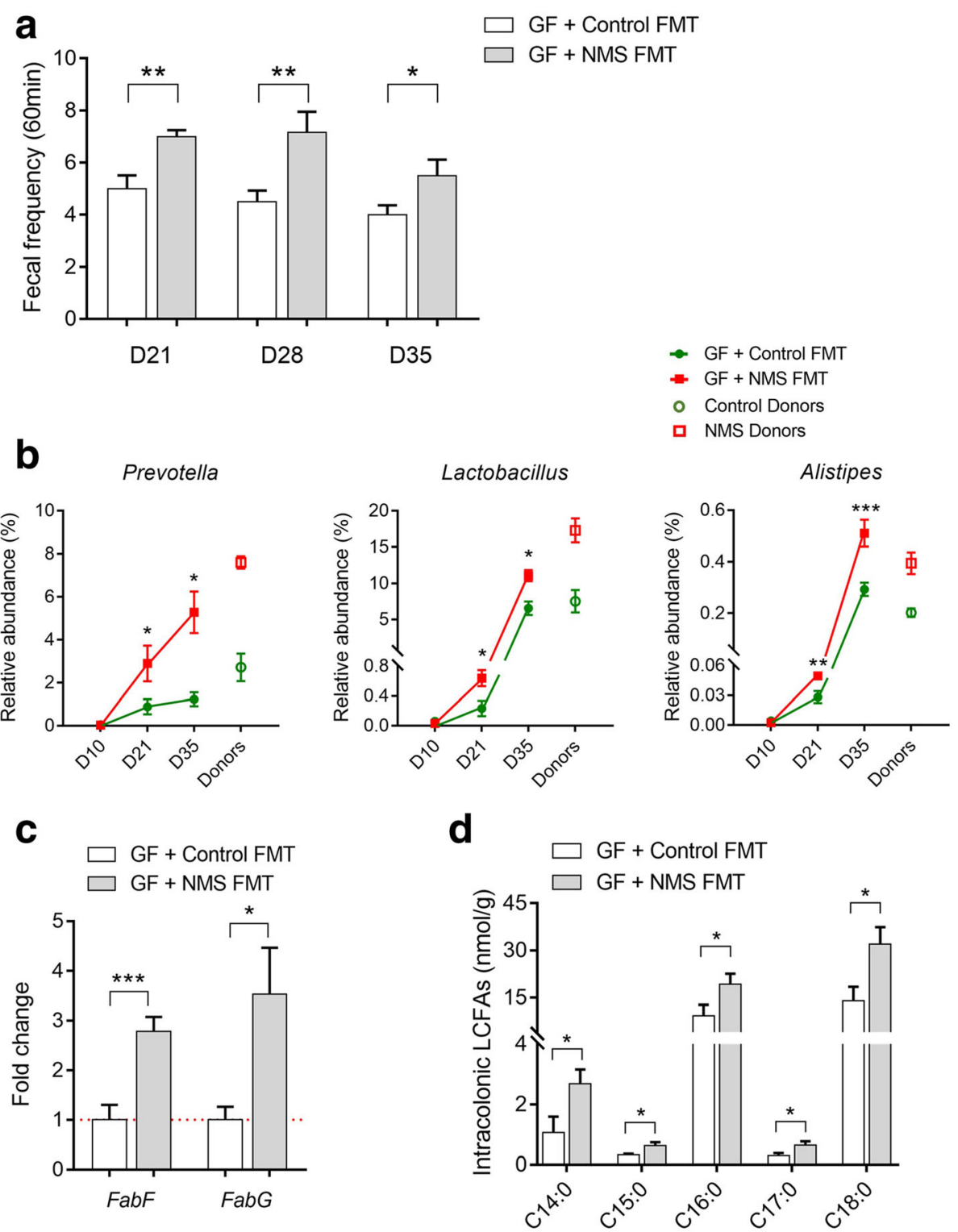

Fig. 5 Fecal microbiota from NMS donors enhances defecation frequency and intracolonic bacterial SLCFAs production in pseudo germ-free (GF) rats ( $n=6$ /group). a Accumulation of fecal pellets within 60 min in colonized GF rats 1 week (day 21), 2 weeks (day 28), and 3 weeks (day 35) after microbial transplant. b Relative abundances of fecal SLCFA-producing genera in donors and colonized GF rats on days 10, 21, and 35. c The relative levels of genes required for SLCFA synthesis in colonic contents of colonized GF rats. $\mathbf{d}$ The amounts of individual SLCFAs per gram of colonic contents. Bar charts are plotted using mean \pm SEM value, and statistical significance between both colonized groups is defined as ${ }^{*} p<$ $0.05 ;{ }^{* *} p<0.01 ;{ }^{* * *} p<0.005$

increased after FMT, and such bacterial profile of GF recipients on day 35 was closer to that of donors (Fig. 5b). Quantitative polymerase chain reaction (qPCR) analysis found that relative levels of two indispensable LCFA-synthesized genes FabF and FabG were increased in the colon lumens of GF rats receiving fecal microbiota of NMS donors on day 35 (Fig. 5c). Consistently, the levels of individual SLCFAs (from C14:0 to C18:0) were obviously raised in the colon lumen (Fig. 5d). These results indicate that featured enterotype of NMS rats could result in elevation of stool frequency and SLCFA generation.

Microbial modulation effectively reduces GI motility and intracolonic SLCFA production in NMS rats treated with neomycin

To further confirm the relationship between SLCFA-producing bacteria and bowel motility, the antibiotic drug neomycin, previously reported as efficient in the modulation of gut dysbiosis and improvement of 
bowel symptoms in patients with IBS [40, 41], was chosen to orally administrate to NMS rats $(150 \mathrm{mg} / \mathrm{kg})$, twice daily, from postnatal days (PD) 56 to 70 (Fig. 1a). Control groups were treated with PBS. Stool frequency was monitored every 3 days during drug intervention. As shown in Fig. 6a, model rats with oral gavage of PBS presented greater stool pellet output within $60 \mathrm{~min}$. Enhanced defecation number was effectively attenuated and ultimately returned to normal after 14 days of neomycin treatment. The gut transit time became prolonged in neomycin-treated NMS rats, but was shorter than control rats (Fig. 6b). Genus-specific PCR analysis found that levels of Prevotella, Lactobacillus, and Alistipes were downregulated in the colon lumens of the neomycin-treated group (Fig. 6c). Also, the levels of SLCFA synthetic genes FabF and FabG were reduced (Fig. 6d). In line with changes of SLCFA-producing bacteria and genes, intracolonic levels of individual SLCFAs were notably attenuated after intervention (Fig. 6e).

\section{Discussion}

In this study, we observed an altered gut microbiota in NMS rats characterized by increased capacity for generation of SLCFAs, which were proved to enhance rat colonic contraction ex vivo and stool frequency in vivo. Further, with microbiota intervention studies including FMT and antibiotic treatment, we found that SLCFA-producing bacteria contribute to the acceleration of colonic motility in rats.

SLCFAs have been reported to regulate GI motility in humans, most of studies concerned their effects on the upper GI tract. A study of 10 healthy men showed that upper intestinal infusion of oils enriched with C18:0 significantly reduced gastric motility [42]. The similar inhibitory impact of SLCFAs on the upper gut motility has also been reported in patients with FGIDs [43]. LCFA-induced slower upper GI motility influences food intake and energy metabolism, demonstrating the importance of SLCFAs in maintenance of body weight as well as in obesity progression [44]. However, we found no difference in the body weight and luminal SLCFAs in the small intestine between NMS rats and controls. A previous study found that increase of defecation was not accompanied by a concomitant change in food consumption in NMS model [45]. Thus, the upper GI motility is possibly not affected by SLCFAs in the NMS model.

The effects of LCFAs on the lower GI tract, i.e., the colon, is another story. Some studies have reported that lipids can increase colon motility in normal subjects and patients with IBS-D [26, 43]. Infusion of unsaturated LCFAs have been shown to accelerate colon transit in human $[46,47]$. But the effects of SLCFAs specifically on the colon motor function have not been reported. This study is the first to provide direct evidence for the promotional action of SLCFAs on colon motility. Compared with SLCFAs, short-chain fatty acids (C3:0 and C4:0) show low potency for stimulating the colonic motor [48]. Also, we found that C2:0, present increased level in fecal VOCs of NMS rats, cannot induce muscle contraction of the isolated colon. This suggests that LCFAs have distinct and specific effects on the colon. Furthermore, elevated muscle contraction induced by C18:0 can be significantly inhibited by a GRP40 antagonist but not a GRP120 antagonist, indicating that GPR40 is specifically involved in the SLCFAs-stimulated colon motility. Briscoe et al. have reported that fatty acids with carbon chain lengths greater than six are able to activate GPR40, thereby giving rise to elevation of calcium secretion and release [49]. Such GPR40-dependent $\mathrm{Ca}^{2+}$ rise can be blocked by inhibition of L-type calcium channels or opening of the K(ATP) channel [50]. These evidences suggest that LCFAs stimulate colon motility possibly through upregulation of GPR40-dependent $\mathrm{Ca}^{2+}$ influx. We intend to explore this possibility in further study.

Generally, luminal SLCFAs are derived from either lipolysis of dietary fats or host/bacterial fatty acid synthesis $[43,51,52]$. A previous study reports normal expression of enzymes for host lipolysis, transport and lipogenesis at the mRNA level in NMS model rats [31]. Moreover, SLCFAs with odd-numbered carbon atoms, such as C15:0 and C17:0, can be produced by bacteria only [32]. If this is true then, elevation of individual SLCFAs from $\mathrm{C} 14$ to $\mathrm{C} 18$ in the colon lumens of NMS model should be the results of gut bacterial actions. In our study, the combination of metabolomic and metagenomic results points to a significant correlation between changes of dominant bacteria and levels of excretive SLCFAs. KO analysis of fecal metagenome showed obvious elevation in the level of fatty acid synthesis and elongation. These findings also support the hypothesis that gut bacteria engage in formation of intracolonic SLCFAs.

Bacterial fatty acid synthesis is essential for supplying the hydrophobic components of the membrane lipids and for providing components of storage lipids [53]. The synthetic process, mainly including initiation and elongation, is executed by a cluster of Fab enzymes that are strongly conserved in bacteria [33, 34, 54]. Of these, FabF and FabG are indispensable for generation of LCFA with carbon atoms 14 to $18[55,56]$. In a previous study of alcoholic liver disease, the gene expression level of FabF and FabG has been determined to reflect the level of bacterial LCFA synthesis in humans and mice [57]. We observed that genomic levels of FabF and FabG were highly enriched in fecal microbiota of NMS rats. Such increased levels of FabF and FabG also appeared in GF rats receiving fecal microbiota from NMS donors, but were attenuated upon modulation of NMS-associated gut dysbiosis. These observations indicate that a higher 


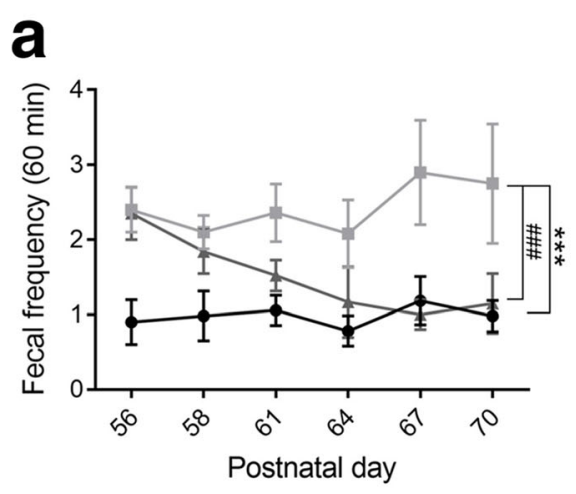

$\square$ Control + PBS
$\square \mathrm{NMS}+$ PBS
$\square \mathrm{NMS}+$ Neomycin

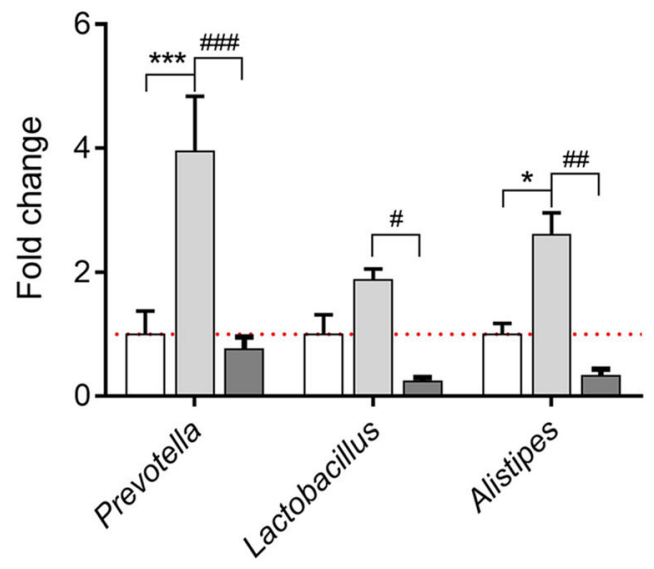

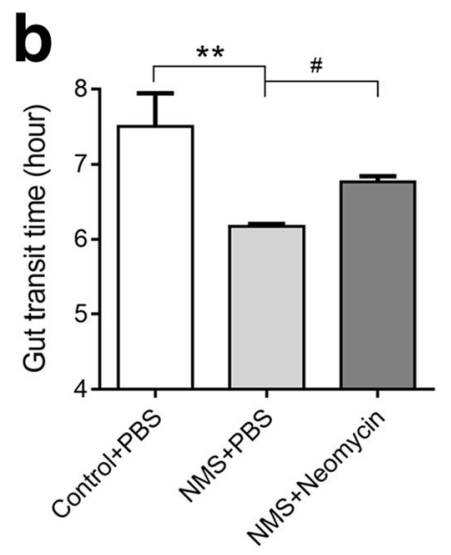

d

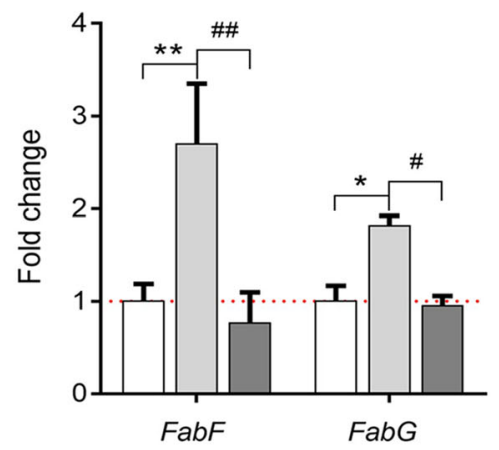

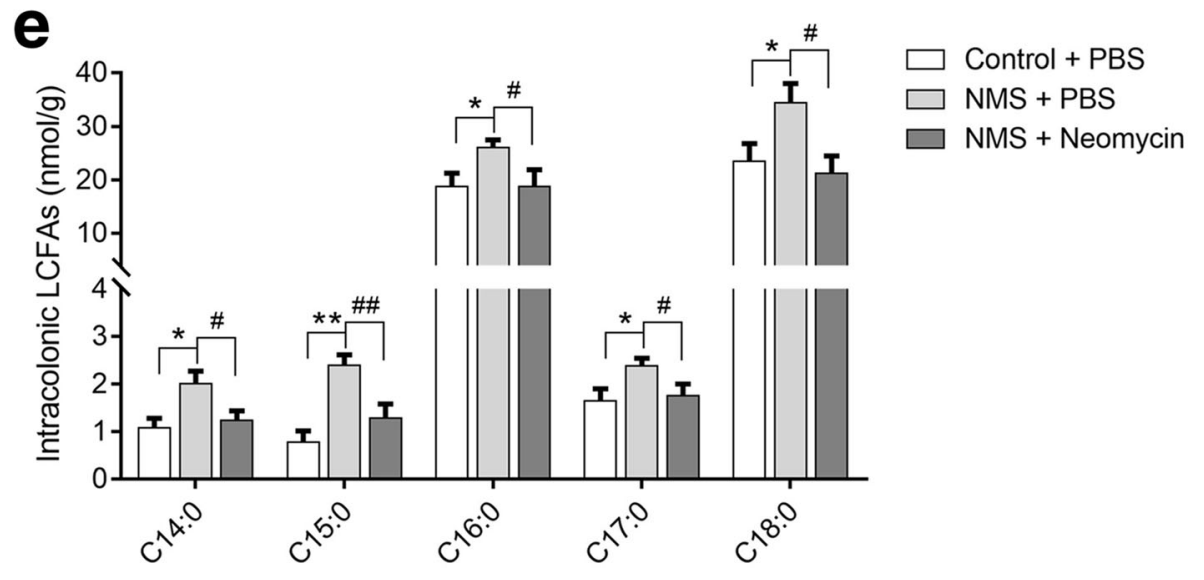

Fig. 6 Modulation of gut microbiota can effectively attenuate bowel motility and intracolonic SLCFAs production in NMS rats based on 14 consecutive days of neomycin treatment $(150 \mathrm{mg} / \mathrm{kg})$. a Accumulation of fecal pellets within 60 min before and after neomycin treatment. $\mathbf{b}$ The gut transit time in rats with or without intervention. c Relative levels of SLCFA-producing genera normalized with 16s rRNA level. d Relative levels of SLCFA-synthetic genes among three groups. e The amounts of individual SLCFAs per gram colonic contents in rats from three groups. Values for line/bar charts are expressed as mean \pm SEM, for box plots are presented as interquartile range. Statistics among three groups was calculated by One-way ANOVA. Significance compared with control group is defined as ${ }^{*} p<0.05$; ${ }^{* *} p<0.01$; ${ }^{* *} p<0.005$, compared with NMS group is described as \#p $<0.05 ; \# \# p<0.01 ; \# \#$; $<0.005$ 
level of bacterial SLCFA synthesis is characteristic of the NMS-associated enterotype.

The genera Prevotella, Lactobacillus and Alistipes expressing essential Fab genes showed elevated abundances in NMS rats and GF rats receiving fecal microbiota of NMS donors, and were significantly reduced in NMS rats after neomycin treatment. Probably, these three genera are important manipulators for intracolonic SLCFA disruption. Interestingly, these SLCFA-producing bacteria have been linked with host GI motility both in clinical and laboratory studies. For example, abundances of Lactobacillus, Prevotella and Alistipes spp. are significantly decreased in patients with constipation [10-12]. Conversely, increase of the genus Lactobacillus has been reported in patients of IBS-D [58]. Higher abundances of Prevotella spp. are found in children with chronic diarrhea [59]. The genera Prevotella, Lactobacillus or Alistipes showed increased abundance in chronic stress-induced rodents characterized by GI dysmotility [24, 25]. Lactobacillus strains accelerate intestinal transit in GF rats and enhance ileal contraction in guinea-pig $[60,61]$. These lines of evidence consistently support a linkage between SLCFA-producing bacteria and host colonic motility; however, further studies are needed to identify the precise roles of specific species or strains in modulating host colonic motility.

By profiling fecal VOCs, we noticed that acidic substances, including SLCFAs and fermented products (e.g., lactate and acetate) were significantly elevated in NMS group, corresponding to the raised acidity in stools. Meanwhile, increase of fecal TCA intermediates citrate and succinate in NMS rats suggest a higher metabolic level of citrate cycle. It is supported by fecal metagenomic results that a significant enrichment of citrate cycle module in NMS group relative controls (different genes mapping in TCA cycle: 398 genes for control and 486 genes for NMS group, adjust $p<0.05)$. Of them, succinate can stimulate water secretion from intestinal segments [62], indicating that increased fecal consistency of NMS rats can be attributed to altered microbial metabolites. In contrast, fecal butyrate and valerate showed reduced levels in the NMS group. Previous study has found that butyrate is beneficial to maintenance of the intestinal epithelial barrier through facilitating tight junction assembly [63]. Soderholm et al. revealed that NMS enhances vulnerability of the colonic mucosal barrier to stress [64]. Probably, deficiency of bacterial butyrate production is one cause of the weak colonic barrier of the NMS model. This evidence indicates the importance of bacterial metabolites in controlling both stool characteristics and colonic barrier function.

\section{Conclusion}

This study determined that NMS rats have excessive levels of intracolonic SLCFAs, and these SLCFAs are specifically related to accelerated colonic movement and increased fecal output. Such elevated SLCFAs is contributed by hyperactive synthetic action of gut bacteria, specifically by the genera Prevotella, Lactobacillus and Alistipes. Fecal microbial transplantation and antibiotic modulation revealed a causal relationship between SLCFA-producing bacteria and host colonic motility. These findings clarify the stimulatory effects of SLCFAs on the colon motility and provide novel insight in gut microbiota-driven GI dysmotility, which may lead to a therapeutic intervention targeting specific gut microbiota for treating GI motility disorders.

\section{Methods \\ Animals}

All Sprague-Dawley (SD) rats used for this study were obtained from the Laboratory Animal Services Centre of The Chinese University of Hong Kong. Rats were maintained on a 12-h light/dark cycle with free access to food and water under specific pathogen-free (SPF) condition. Materials including cages, diets, water, and litters were sterilized, and stool frequency was tested in a clean hood with a UV lamp for pseudo GF rats. All animal experiments have followed the Animals Ordinance, Department of Health, Hong Kong SAR, China.

\section{Neonatal maternal separation modeling and colonic motility assessment}

Referring to our previous study [65], the procedure of the NMS model was performed as shown in the timeline (Fig. 1a). Briefly, four pregnant SD rats were housed individually in cages. Randomly, newborn pups from two mothers were assigned to NMS group and pups from another two dams were assigned to non-handled control group. NMS pups were separated from their dams during the period of postnatal days (PD) 2 to 14 for $3 \mathrm{~h}$ daily whereas control pups remained with their mothers constantly. All pups were weaned on PD 22, and only male pups ( $n=8$ /group) with similar body weight was used for further experiments. GI motility was individually evaluated in rats 8 weeks later. Fecal pellets were collected to measure $\mathrm{pH}$ level and water contents, and were used for analyses of metagenomics and metabolomics. The detail methods for assessment of motor function and stool characteristics were described in Additional file 1. The luminal contents of ileum $(10 \mathrm{~cm}$ proximal to the cecum), cecum, and proximal colon ( $5 \mathrm{~cm}$ distal to the cecum) were collected after $\mathrm{CO}_{2}$ anesthesia, and were immediately stored at $-80{ }^{\circ} \mathrm{C}$. Furthermore, to investigate the relationship between SLCFA-produced bacteria and the colonic motor in NMS rats, the drug neomycin $(150 \mathrm{mg} / \mathrm{kg})$ was twice daily administrated to adult NMS rats for 14 consecutive 
days (from PD 56 to 70). Meanwhile, other NMS or control rats were orally gavaged with PBS.

\section{Pseudo germ-free modeling and fecal microbial transfer} The experimental procedure is shown in Fig. 1b. The antibiotic cocktail (ABX), containing ampicillin (1 g/L), neomycin $(1 \mathrm{~g} / \mathrm{L})$, and metronidazole $(0.5 \mathrm{~g} / \mathrm{L})$ were prepared in drinking water as previously described [37]. The ABX-contained water was ad libitum supplied for 12 normal SD rats ( $n=6 /$ group) to establish the pseudo GF model. No difference in the record of daily and total water consumption (Additional file 1: Fig. S4A) indicates that rats in different cages ingested similar volumes of ABX. To ensure elimination of the gut microbiome, fecal pellets were frequently collected during experiment for real-time monitoring of dynamic changes of gut microbiota. The very low levels of fecal total DNA quality and bacterial diversity (Additional file 1: Fig. S4B and C) indicate that the pseudo GF model was successfully established after 10 days of ABX-water intervention. Furthermore, donors' feces from either NMS or control group ( $n=8$ /group) were individually pooled and completely homogenized in pre-reduced PBS at $1 \mathrm{ml}$ per pellet referring to a published method [20]. One milliliter of the settled suspension was daily administered to pseudo GF rats for five consecutive days. The stool frequency was weekly determined in colonized GF rats. Fecal samples were collected on Day 0, 10, 21 and 35 for further microbial analysis, and colonic contents were collected on Day 35 for assessment of SLCFA producing genes and SLCFA amounts.

\section{Preparation of saturated long-chain fatty acids for colonic motility assessments}

To evaluate the effects of SLCFAs on host colonic motility, dominant odd- and even-numbered carbon SLCFAs C17:0 and C18:0 were chosen to individually test their influences on the muscle contraction of rat isolated colon segments ex vivo and rat stool frequency in vivo. C17:0 and C18:0 (Cat\#: 506-12-7 and 57-11-4, Sigma-Aldrich, St. Louis, MO USA) were solubilized with fatty acid free-bovine serum albumin (BSA, Cat\#: 9048-46-8; Sigma-Aldrich, USA) to generate a series of concentrations $(10,30,50$ and $100 \mu \mathrm{M})$ for organ bath system-based colonic contraction test ex vivo [66]. The concentration range were calculated in accordance with SLCFA levels found in the colon contents of NMS rats with $1.1 \mathrm{~g} / \mathrm{cm} 3$ of density of the colonic contents reported in a previous study [67]. To test which long-chain fatty acid receptors mediate SLCFA-stimulated colonic contraction, selective GPR40 antagonist DC260126 (DC, Cat\#: 346692-04-4, Tocris Bioscience, UK) and GPR120 antagonist AH7614 (AH, Cat\#: 6326-06-3, Tocris Bioscience, UK) was prepared at working dosages of $2.5,5$, and $10 \mu \mathrm{M}$ according to previous studies $[68,69]$. Acetylcholine (Ach, Cat\#: 60-31-1, Sigma, St. Louis, MO USA) and C2:0 (Cat\#: 127-09-3, Sigma, St. Louis, MO USA) were applied as positive and negative control, respectively. The experimental procedure was described in Additional file 1. Furthermore, both SLCFAs were prepared as different dosages (1, 2.5 and $5 \mathrm{mg} / \mathrm{kg}$, dissolved in $5 \%$ ethanol) for in vivo measurement of stool frequency in normal SD rats ( $n=8$ /group). Each rat orally treated with either $1 \mathrm{~mL}$ of SLCFAs or vehicle solution was located in an individual cage for defecation frequency assessment. Fecal pellets were collected for determination of intracolonic SLCFA levels.

\section{Total DNA isolation and metagenomic sequencing}

Total bacterial DNA was isolated and purified from fecal pellets (precisely weighted $200 \mathrm{mg}$ ) using a stool DNA Isolation Kit (Qiagen, Valencia, CA). All samples were sequenced based on the Illumina Hiseq 4000 platform (paired-end; insert size, $350 \mathrm{bp}$; read length, $150 \mathrm{bp}$ ). After removal of adaptor and low-quality reads, the remaining reads were filtered to eliminate the host DNA genome based on the genome reference of Rattus norvegicus by SOAPalign v2.21 [70]. Finally, $105.81 \mathrm{~Gb}$ high-quality pair-end reads for the 16 rats samples $(n=$ 8/group) was acquired with an average of $6.61 \mathrm{~Gb}$ per sample in groups of NMS and control (Additional file 2: Table S2).

\section{Construction of the gene catalog}

The reads were assembled into contigs for all samples using the assembly software SOAPdenovo v2.0455. Average $75.67 \%$ of the total reads were used to generate 1.6 million contigs without ambiguous bases (minimum length of $500 \mathrm{bp}$ ). ORFs were predicted from the assembled contigs using the MetaGeneMark v3.26 programme [71]. The 6,116,823 ORFs longer than 100 bp covered $89.67 \%$ of the total length of the contigs and about half (50.51\%) of the ORFs appeared complete. All ORFs were clustered by CD-hit v4.6.4 to construct a non-redundant gene catalog using a stringent criterion of $95 \%$ identity at the nucleotide level over $90 \%$ of the length of the shorter ORFs [72]. The final non-redundant gene set contained 1,462,418 ORFs with an average length of 728 bp.

\section{Diversity analysis, taxonomic assignment and functional characterization}

The $\alpha$-diversity (within-sample diversity) and $\beta$-diversity (between-sample diversity) were estimated by the Shannon index and Bray-Curtis dissimilarity metric, respectively [73]. For taxonomic assignment, all predicted genes were blasted against the reference microbial genomes from NCBI (including 4258 microbial genomes) by using 
BLAST (v 2.2.26, default parameter) with at least $80 \%$ overlap of query. Taxonomic identification was performed as $65 \%$ identity for phylum, $85 \%$ identity for genus, 95\% identity for species $[74,75]$. The taxonomic abundance was calculated based on gene abundance, and protein sequences of the predicted genes were searched using National Center for Biotechnology Information BLASTP against the KEGG gene database (v79). Each protein was assigned to the KEGG group by the highest scoring annotated hits. Significance in the relative abundance of genes, KOs, phylum, genera, and species between both groups were compared by two-tailed Wilcoxon rank-sum test (Additional file 2: Tables S3-S7). The relationship between the abundance of each species and SLCFAs contents was assessed by the Spearman's correlation [76]. Enrichment in NMS or control group was then determined according to the higher rank-sum.

\section{Determination of bacterial DNA quality and $16 \mathrm{~s}$ ribosomal RNA amplicon sequencing}

DNA extracts was prepared from feces (precisely weighted $200 \mathrm{mg}$ ) of donors and colonized GF rats. The DNA yield and quality were determined spectrophotometrically by the NanoDrop ${ }^{\mathrm{TM}}$ ND-2000 (Thermo Fisher Scientific Ltd., Waltham, MA, USA). The DNA integrity was determined through $1 \%$ agarose gel $(w / v)$. Furthermore, the extracted DNA (30 ng) was amplified with universal primers (515F and 806R) to obtain the V4 regions of the $16 \mathrm{~S}$ rRNA gene. The PCR products were purified and sequenced on the Illumina Hiseq 2500 platform. After raw data filtering and merging [77, 78], the numbers of tag and operational taxonomic unit (OTU) were generated from 50 samples (Additional file 2: Table S8). Bacterial diversity and taxonomy was obtained from OTU table by using QIIME software package [79].

\section{PCR analysis of genus-specific bacterial 16s rDNA and SLCFA synthetic genes}

Genus-specific primers for Prevotella, Lactobacillus and Alistipes were obtained from published studies [32, 80]. Bacterial DNA extracts (50 ng) of colonic contents were amplified with pairs of primers and Power SYBR GREEN Master Mix (Applied Biosystems, Foster city, CA, USA) based on an ABI StepOne Plus Sequence Detection System (Applied Biosystems, Foster city, CA, USA). The cycling conditions were as follows: $95{ }^{\circ} \mathrm{C}$ for $10 \mathrm{~min}$, followed by 40 cycles of $95{ }^{\circ} \mathrm{C}$ for $30 \mathrm{~s}, 52{ }^{\circ} \mathrm{C}$ for $30 \mathrm{~s}$, and $72{ }^{\circ} \mathrm{C}$ for $1 \mathrm{~min}$. Moreover, such SYBR GREEN-based PCR detection also applied to testify expression of LCFA synthetic FabF and FabG genes from colonic contents. The primers for FabF and FabG were designed as previously described [57]. The expression of each genus and gene was normalized to the level of $16 \mathrm{~S}$ rRNA.

\section{Metabolite extraction and SLCFA quantification}

Fecal samples or luminal contents (100 mg) were completely homogenized with five-fold volume of ice-cold distilled water. After high-speed centrifugation $\left(13,000 \mathrm{rpm}\right.$ for $15 \mathrm{~min}$ at $\left.4{ }^{\circ} \mathrm{C}\right)$, water extractions were transferred to a new 2-mL tube. Subsequently, a five-fold volume $(500 \mu \mathrm{L})$ of methanol was added into the pellet sample. The mixture was completely homogenized and centrifuged again. Methanol extractions were combined with the previous water extractions. Strongly vortex and centrifugation again, the resulting supernatants were obtained for derivatization processing. Meanwhile, a quality control (QC) sample pooling all rat samples was prepared using the same protocol. For quantification of luminal SFAs in the small and large intestines, external calibration solution of SFA standards from C2:0 to C18:0 were prepared at a series of concentrations (from 1 to $20,000 \mathrm{ng} / \mathrm{ml}$ ). Isotope labeled $\mathrm{C}_{13}$-myristic acid was used as internal standards. Absolute quantities of individual SFAs were normalized to the sample weight.

\section{GC/MS-based metabolomic analysis and data processing}

Fecal metabolites were derivatized by BSTFA with $1 \%$ TMCS based on a previously method [81]. A gas chromatography coupled with a mass spectrum (GCMS-QP2010 systems, Shimaduzu Co., Tokyo, Japan) was applied for fecal metabolome analysis. Fecal derivatives were separated by a DB-5 MS fused-silica capillary column (30 m $\times$ $250 \mu \mathrm{m}$ i.d.; Agilent J\&W Scientific, Folsom, CA), chemically bonded with a 5\% phenyl-95\% methylpolysiloxane cross-linked stationary phase $(0.25 \mu \mathrm{m}$ film thickness). The detail sample preparation and analytical parameters of instrumental conditions were showed in Additional file 1 . The metabolic signals were deconvoluted, aligned and normalized to final data matrix through using $\mathrm{R}$-scipt with $\mathrm{xcms}$ package ( $\mathrm{R}$ version 3.4 .2 ). The processed matrix was introduced into the software SIMCA-P (Version 11.0, Umetrics, Umea, Sweden) for principal component analysis (PCA) and partial least squares-discriminant analysis (PLS-DA). Metabolic features were selected by combination of the variable importance in the projection (VIP) threshold (VIP > 1.0) and the Student's $t$-test $(p<0.05)$. Metabolites were identified by the national institute of standards and technology (NIST) library (over 95\% matched similarity).

\section{Statistical analysis}

For sequencing data, statistical analyses were performed in $\mathrm{R}$ (v3.4.10) software; differential abundance of genes, taxonomies, and KOs were evaluated by two-tailed 
Wilcoxon rank-sum test. For determination of metabolites, genes, and colonic motor in animal studies, Mann-Whitney test was used for statistics between two groups, and one-way ANOVA was used for comparison among more than two groups. These statistical analyses were performed in GraphPad Prism 6 (GraphPad software lnc., CA, USA). Statistical significance is defined as $p<0.05$.

\section{Additional files}

Additional file 1 Figure S1. Fecal characteristics and body weight in NMS rats and controls. Figure S2. Muscle amplitudes of rat isolated colonic segments with different treatment. Figure S3. Phylogenetic profiles of fecal microbiomes of NMS and control rats. Figure S4. Fecal microbial community in pseudo GF rats at duration of FMT experiment. Table S1. Fecal metabolites with significant difference between NMS rats and controls. (DOCX $784 \mathrm{~kb})$

Additional file 2 Table S2. Data production, quality control, assembly result and gene prediction resulted from fecal metagenomic sequencing analysis. Table S3. Taxonomic profiles of fecal microbiota in NMS and control groups at phylum level. Table S4. Taxonomic profiles of fecal microbiota in NMS and control groups at genus level. Table S5. Taxonomic profiles of fecal microbiota in NMS and control groups at species level. Table S6. Correlation between species and saturated longchain fatty acids determined by fecal metagenomic and metabolomic analyses. Table S7. Identified KOs involved in fatty acid synthesis and degradation. Table S8. Data production and quality control of fecal samples from 16s rRNA amplicon sequencing analysis. Table S9. Taxonomic changes at genus level between colonized GF rats and donors. (XLSX $73 \mathrm{~kb}$ )

\section{Abbreviations}

ABX: Antibiotic cocktails; Acaca: Acetyl-CoA carboxylase; C14:0: Tetradecanoic acid; C15:0: Pentadecanoic acid; C16:0: Palmitic acid; C17:0: Heptadecanoic acid; C18:0: Octadecanoic acid; C2:0: Acetic acid; C4:0: Butyric acid; C5:0: Valeric acid; FabD: Malonyl CoA-acyl carrier protein; FabF: 3-Oxoacyl[acyl-carrier-protein] synthase II; FabG: 3-Oxoacyl-[acyl-carrier protein] reductase; Fabl: Enoyl-[acyl-carrier protein] reductase I; FabZ: 3-Hydroxyacyl[acyl-carrier-protein] dehydratase; FadA: Acetyl-CoA acyltransferase; FadB: 3Hydroxybutyryl-CoA dehydrogenase; FadD: Long-chain acyl-CoA synthetase; FadE: Acyl-CoA dehydrogenase; FadH: 2,4-Dienoyl-CoA reductase; FadL: Long-chain fatty acid transport protein; Fas: Fatty acid synthase (bacteria type); NMS: Neonatal maternal separation; PCoA: Principal coordinate analysis; SLCFAs: Saturated long-chain fatty acids; TesA: Acyl-CoA thioesterase I; VIP: Variable importance in the projection; VOCs: Volatile organic compounds; YciA: Acyl-CoA 12 thioesterase

\section{Acknowledgments}

We thank the colleagues of Shenzhen Key Laboratory of Human Commensal Microorganisms and Health Research and Shenzhen Engineering Laboratory of Detection and Intervention of Human Intestinal Microbiome for their assistance in microbial sequencing and analysis. We also thank Dr. Martha Dahlen (CA, USA) for her critical appraisal for this manuscript.

\section{Funding}

This project was supported by grants from Faculty Research Grant of Hong Kong Baptist University (FRG2/15-16/001 and FRG2/16-17/003), the Research Grants Council of Hong Kong Collaborative Research Fund (C2012-15G), and Guangdong-Hong Kong Technology Cooperation Funding Scheme (2016A050503039).

\section{Availability of data and materials}

Fecal metagenomic sequencing reads are deposited in the National Center for Biotechnology Information (NCBI) database with accession code PRJNA419985. Other data that support the findings of this study are available from the corresponding author upon reasonable request.

\section{Authors' contributions}

$Z X B, X D F, L X Z$, and $L Z$ conceived the idea and designed this study. $L Z$ and LL performed metabolomics, in vivo and ex vivo experiments using animals. YF and YC contributed to metagenomic and 16s rRNA sequencing analysis. WY and ZSL conducted bacterial DNA extraction and relevant PCR analysis. $L Z, T H, C Y L$, and HLW prepared manuscript. HMY, JW, and LXZ provided guidance for both experimental design and manuscript preparation. All authors read and approved the final manuscript.

\section{Ethics approval}

The use and care of all animals were performed in accordance with the Animals Ordinance, and experimental operators obtained licenses issued by the government of the Hong Kong Special Administrative Region Department of Health, Hong Kong SAR, China.

\section{Competing interests}

The authors declare that they have no competing interests.

\section{Publisher's Note}

Springer Nature remains neutral with regard to jurisdictional claims in published maps and institutional affiliations.

\section{Author details}

${ }^{1}$ Chinese Medicine Clinical Study Center, Jockey Club School of Chinese Medicine, Hong Kong Baptist University, Kowloon Tong, Hong Kong SAR, China. ${ }^{2} \mathrm{BGI}$ Genomics, BGI-Shenzhen, Shenzhen, China. ${ }^{3}$ Preparatory Office of Shenzhen-Melbourne Institute of Life Sciences and Bioengineering, Guangzhou University of Chinese Medicine, Guangzhou, China. ${ }^{4}$ YMU-HKBU Joint Laboratory of Traditional Natural Medicine, Yunnan Minzu University, Kunming, China. ${ }^{5}$ The Second Affiliated Hospital of Guangzhou University of Chinese Medicine, Guangzhou, China. ${ }^{6} \mathrm{BGl}$-Shenzhen, Shenzhen, China. ${ }^{7}$ Digestive Diseases and Nutrition Center, Department of Pediatrics, The State University of New York at Buffalo, 3435 Main Street, 422BRB, Buffalo, NY 14214, USA.

Received: 13 December 2017 Accepted: 1 June 2018

Published online: 14 June 2018

\section{References}

1. Corazziari E. Definition and epidemiology of functional gastrointestinal disorders. Best Pract Res Clin Gastroenterol. 2004;18:613-31.

2. Bassotti G, Antonelli E, Villanacci V, Salemme M, Coppola M, Annese V. Gastrointestinal motility disorders in inflammatory bowel diseases. World J Gastroenterol. 2014;20:37-44.

3. Lacy BE, Weiser K. Gastrointestinal motility disorders: an update. Dig Dis. 2006:24:228-42

4. Ohama T, Hori M, Ozaki H. Mechanism of abnormal intestinal motility in inflammatory bowel disease: how smooth muscle contraction is reduced? J Smooth Muscle Res. 2007;43:43-54.

5. Beyder A, Farrugia G. Targeting ion channels for the treatment of gastrointestinal motility disorders. Therap Adv Gastroenterol. 2012;5:5-21.

6. Hansen MB. Neurohumoral control of gastrointestinal motility. Physiol Res. 2003:52:1-30.

7. Quigley EM. Microflora modulation of motility. J Neurogastroenterol Motil. 2011;17:140-7.

8. Matto J, Maunuksela L, Kajander K, Palva A, Korpela R, Kassinen A, Saarela M. Composition and temporal stability of gastrointestinal microbiota in irritable bowel syndrome-a longitudinal study in IBS and control subjects. FEMS Immunol Med Microbiol. 2005;43:213-22.

9. Pozuelo M, Panda S, Santiago A, Mendez S, Accarino A, Santos J, Guarner F, Azpiroz F, Manichanh C. Reduction of butyrate- and methane-producing microorganisms in patients with irritable bowel syndrome. Sci Rep. 2015;5:12693.

10. Khalif IL, Quigley EM, Konovitch EA, Maximova ID. Alterations in the colonic flora and intestinal permeability and evidence of immune activation in chronic constipation. Dig Liver Dis. 2005;37:838-49.

11. Zhu L, Liu W, Alkhouri R, Baker RD, Bard JE, Quigley EM, Baker SS. Structural changes in the gut microbiome of constipated patients. Physiol Genomics. 2014;46:679-86. 
12. de Meij TG, de Groot EF, Eck A, Budding AE, Kneepkens CM, Benninga MA, van Bodegraven AA, Savelkoul PH. Characterization of microbiota in children with chronic functional constipation. PLoS One. 2016;11:e0164731.

13. Kim SE, Choi SC, Park KS, Park MI, Shin JE, Lee TH, Jung KW, Koo HS, Myung SJ. Constipation research group of Korean Society of N, motility: change of fecal Flora and Effectiveness of the short-term VSL\#3 probiotic treatment in patients with functional constipation. J Neurogastroenterol Motil. 2015;21:111-20.

14. De Palma G, Lynch MD, Lu J, Dang VT, Deng Y, Jury J, Umeh G, Miranda PM, Pigrau Pastor M, Sidani S, et al. Transplantation of fecal microbiota from patients with irritable bowel syndrome alters gut function and behavior in recipient mice. Sci Transl Med. 2017;9 https:// doi.org/10.1126/scitransImed.aaf6397.

15. Ge X, Zhao W, Ding C, Tian H, Xu L, Wang H, Ni L, Jiang J, Gong J, Zhu W, et al. Potential role of fecal microbiota from patients with slow transit constipation in the regulation of gastrointestinal motility. Sci Rep. 2017;7:441.

16. Barbara G, Stanghellini V, Brandi G, Cremon C, Di Nardo G, De Giorgio R, Corinaldesi R. Interactions between commensal bacteria and gut sensorimotor function in health and disease. Am J Gastroenterol. 2005;100:2560-8.

17. Triantafyllou K, Chang C, Pimentel M. Methanogens, methane and gastrointestinal motility. J Neurogastroenterol Motil. 2014;20:31-40.

18. Anitha M, Vijay-Kumar M, Sitaraman SV, Gewirtz AT, Srinivasan S. Gut microbial products regulate murine gastrointestinal motility via toll-like receptor 4 signaling. Gastroenterology. 2012;143:1006-1016 e1004.

19. Cherbut $C$. Motor effects of short-chain fatty acids and lactate in the gastrointestinal tract. Proc Nutr Soc. 2003;62:95-9.

20. Yano JM, Yu K, Donaldson GP, Shastri GG, Ann P, Ma L, Nagler CR, Ismagilov RF, Mazmanian SK, Hsiao EY. Indigenous bacteria from the gut microbiota regulate host serotonin biosynthesis. Cell. 2015;161:264-76.

21. Bulbul M, Babygirija R, Cerjak D, Yoshimoto S, Ludwig K, Takahashi T. Impaired adaptation of gastrointestinal motility following chronic stress in maternally separated rats. Am J Physiol Gastrointest Liver Physiol. 2012;302:G702-11.

22. Zhang $M$, Leung $F P$, Huang $Y$, Bian ZX. Increased colonic motility in a rat model of irritable bowel syndrome is associated with up-regulation of $\mathrm{L}$ type calcium channels in colonic smooth muscle cells. Neurogastroenterol Motil. 2010;22:e162-70.

23. Bian ZX, Zhang M, Han QB, Xu HX, Sung JJ. Analgesic effects of JCM-16021 on neonatal maternal separation-induced visceral pain in rats. World $J$ Gastroenterol. 2010;16:837-45.

24. De Palma G, Blennerhassett P, Lu J, Deng Y, Park AJ, Green W, Denou E, Silva MA, Santacruz A, Sanz Y, et al. Microbiota and host determinants of behavioural phenotype in maternally separated mice. Nat Commun. 2015;6:7735.

25. Pusceddu MM, El Aidy S, Crispie F, O'Sullivan O, Cotter P, Stanton C, Kelly P, Cryan JF, Dinan TG. N-3 polyunsaturated fatty acids (PUFAs) reverse the impact of early-life stress on the gut microbiota. PLoS One. 2015;10:e0139721.

26. Deiteren A, Camilleri M, Burton D, McKinzie S, Rao A, Zinsmeister AR. Effect of meal ingestion on ileocolonic and colonic transit in health and irritable bowel syndrome. Dig Dis Sci. 2010;55:384-91.

27. Lin CY, Zhang M, Huang T, Yang LL, Fu HB, Zhao L, Zhong LL, Mu HX, Shi $X K$, Leung CF, et al. Spexin enhances bowel movement through activating L-type voltage-dependent Calcium Channel via Galanin receptor 2 in mice. Sci Rep. 2015:5:12095.

28. Edfalk S, Steneberg P, Edlund H. Gpr40 is expressed in enteroendocrine cells and mediates free fatty acid stimulation of incretin secretion. Diabetes 2008:57:2280-7.

29. Paulsen SJ, Larsen LK, Hansen G, Chelur S, Larsen PJ, Vrang N. Expression of the fatty acid receptor GPR120 in the gut of diet-induced-obese rats and its role in GLP-1 secretion. PLoS One. 2014;9:e88227.

30. Ichimura A, Hirasawa A, Hara T, Tsujimoto G. Free fatty acid receptors act as nutrient sensors to regulate energy homeostasis. Prostaglandins Other Lipid Mediat. 2009;89:82-8.

31. Paternain L, Martisova E, Milagro Fl, Ramirez MJ, Martinez JA, Campion J. Postnatal maternal separation modifies the response to an obesogenic diet in adulthood in rats. Dis Model Mech. 2012;5:691-7.

32. Kumagai H, Maisawa S, Tanaka M, Takahashi M, Takasago Y, Nishijima A, Watanabe S. Intestinal microbiota and secretory immunoglobulin a in feces of exclusively breast-fed infants with blood-streaked stools. Microbiol Immunol. 2012;56:657-63.

33. Qiu X, Choudhry AE, Janson CA, Grooms M, Daines RA, Lonsdale JT, Khandekar SS. Crystal structure and substrate specificity of the beta- ketoacyl-acyl carrier protein synthase III (FabH) from Staphylococcus aureus. Protein Sci. 2005;14:2087-94.

34. Cronan JE, Thomas J. Bacterial fatty acid synthesis and its relationships with polyketide synthetic pathways. Methods Enzymol. 2009:459:395-433.

35. Schweizer E, Hofmann J. Microbial type I fatty acid synthases (FAS): major players in a network of cellular FAS systems. Microbiol Mol Biol Rev. 2004;68: 501-17. table of contents

36. Dellomonaco C, Clomburg JM, Miller EN, Gonzalez R. Engineered reversal of the beta-oxidation cycle for the synthesis of fuels and chemicals. Nature 2011:476:355-9.

37. Ijssennagger N, Belzer C, Hooiveld GJ, Dekker J, van Mil SWC, Muller M, Kleerebezem M, van der Meer R. Gut microbiota facilitates dietary hemeinduced epithelial hyperproliferation by opening the mucus barrier in colon. Proc Natl Acad Sci U S A. 2015;112:10038-43.

38. Nicklas W, Keubler L, Bleich A. Maintaining and monitoring the defined microbiota status of Gnotobiotic rodents. ILAR J. 2015;56:241-9.

39. Ge X, Ding C, Zhao W, Xu L, Tian H, Gong J, Zhu M, Li J, Li N. Antibioticsinduced depletion of mice microbiota induces changes in host serotonin biosynthesis and intestinal motility. J Transl Med. 2017;15:13.

40. Basseri RJ, Weitsman S, Barlow GM, Pimentel M. Antibiotics for the treatment of irritable bowel syndrome. Gastroenterol Hepatol (N Y). 2011;7:455-93.

41. Ahmad OF, Akbar A. Microbiome, antibiotics and irritable bowel syndrome. Br Med Bull. 2016;120:91-9.

42. French SJ, Conlon CA, Mutuma ST, Arnold M, Read NW, Meijer G, Francis J. The effects of intestinal infusion of long-chain fatty acids on food intake in humans. Gastroenterology. 2000;119:943-8.

43. Feinle-Bisset C, Azpiroz F. Dietary lipids and functional gastrointestinal disorders. Am J Gastroenterol. 2013;108:737-47.

44. Matzinger D, Degen L, Drewe J, Meuli J, Duebendorfer R, Ruckstuhl N, D'Amato $M$, Rovati L, Beglinger $C$. The role of long chain fatty acids in regulating food intake and cholecystokinin release in humans. Gut. 2000;46:688-93.

45. Coutinho SV, Plotsky PM, Sablad M, Miller JC, Zhou H, Bayati Al, McRoberts JA, Mayer EA. Neonatal maternal separation alters stress-induced responses to viscerosomatic nociceptive stimuli in rat. Am J Physiol Gastrointest Liver Physiol. 2002;282:G307-16.

46. Spiller RC, Brown ML, Phillips SF. Decreased fluid tolerance, accelerated transit, and abnormal motility of the human colon induced by oleic acid. Gastroenterology. 1986;91:100-7.

47. Kamath PS, Phillips SF, O'Connor MK, Brown ML, Zinsmeister AR. Colonic capacitance and transit in man: modulation by luminal contents and drugs. Gut. 1990;31:443-9.

48. Hurst NR, Kendig DM, Murthy KS, Grider JR. The short chain fatty acids, butyrate and propionate, have differential effects on the motility of the Guinea pig colon. Neurogastroenterol Motil. 2014;26:1586-96.

49. Briscoe CP, Tadayyon M, Andrews JL, Benson WG, Chambers JK, Eilert MM Ellis C, Elshourbagy NA, Goetz AS, Minnick DT, et al. The orphan G proteincoupled receptor GPR40 is activated by medium and long chain fatty acids. J Biol Chem. 2003;278:11303-11.

50. Shapiro H, Shachar S, Sekler I, Hershfinkel M, Walker MD. Role of GPR40 in fatty acid action on the beta cell line INS-1E. Biochem Biophys Res Commun. 2005:335:97-104.

51. Niot I, Poirier H, Tran TT, Besnard P. Intestinal absorption of long-chain fatty acids: evidence and uncertainties. Prog Lipid Res. 2009;48:101-15.

52. Smith S, Witkowski A, Joshi AK. Structural and functional organization of the animal fatty acid synthase. Prog Lipid Res. 2003;42:289-317.

53. Parsons JB, Rock CO. Bacterial lipids: metabolism and membrane homeostasis. Prog Lipid Res. 2013;52:249-76.

54. Heath RJ, Rock CO. Enoyl-acyl carrier protein reductase (fabl) plays a determinant role in completing cycles of fatty acid elongation in Escherichia coli. J Biol Chem. 1995;270:26538-42.

55. Jeon E, Lee S, Lee S, Han SO, Yoon YJ, Lee J. Improved production of longchain fatty acid in Escherichia coli by an engineering elongation cycle during fatty acid synthesis (FAS) through genetic manipulation. J Microbiol Biotechnol. 2012;22:990-9.

56. Garwin JL, Klages AL, Cronan JE Jr. Beta-ketoacyl-acyl carrier protein synthase II of Escherichia coli. Evidence for function in the thermal regulation of fatty acid synthesis. J Biol Chem. 1980;255:3263-5.

57. Chen P, Torralba M, Tan J, Embree M, Zengler K, Starkel P, van Pijkeren JP, DePew J, Loomba R, Ho SB, et al: Supplementation of saturated long-chain fatty acids maintains intestinal eubiosis and reduces ethanol-induced liver injury in mice. Gastroenterology 2015, 148:203-214 e216. 
58. Carroll IM, Chang YH, Park J, Sartor RB, Ringel Y. Luminal and mucosalassociated intestinal microbiota in patients with diarrhea-predominant irritable bowel syndrome. Gut Pathog. 2010;2:19.

59. Gilchrist CA, Petri SE, Schneider BN, Reichman DJ, Jiang N, Begum S, Watanabe K, Jansen CS, Elliott KP, Burgess SL, et al. Role of the gut microbiota of children in diarrhea due to the protozoan parasite Entamoeba histolytica. J Infect Dis. 2016;213:1579-85.

60. Husebye E, Hellstrom PM, Sundler F, Chen J, Midtvedt T. Influence of microbial species on small intestinal myoelectric activity and transit in germ-free rats. Am J Physiol Gastrointest Liver Physiol. 2001;280:G368-80.

61. Massi M, loan P, Budriesi R, Chiarini A, Vitali B, Lammers KM, Gionchetti P, Campieri M, Lembo A, Brigidi P. Effects of probiotic bacteria on gastrointestinal motility in Guinea-pig isolated tissue. World J Gastroenterol. 2006;12:5987-94

62. Inagaki A, Ichikawa H, Sakata T. Inhibitory effect of succinic acid on epithelial cell proliferation of colonic mucosa in rats. J Nutr Sci Vitaminol (Tokyo). 2007;53:377-9.

63. Peng L, Li ZR, Green RS, Holzman IR, Lin J. Butyrate enhances the intestinal barrier by facilitating tight junction assembly via activation of AMP-activated protein kinase in Caco-2 cell monolayers. J Nutr. 2009;139:1619-25.

64. Soderholm JD, Yates DA, Gareau MG, Yang PC, MacQueen G, Perdue MH. Neonatal maternal separation predisposes adult rats to colonic barrier dysfunction in response to mild stress. Am J Physiol Gastrointest Liver Physiol. 2002;283:G1257-63.

65. Chung EK, Zhang XJ, Xu HX, Sung JJ, Bian ZX. Visceral hyperalgesia induced by neonatal maternal separation is associated with nerve growth factor-mediated central neuronal plasticity in rat spinal cord. Neuroscience. 2007;149:685-95.

66. Huang S, Rutkowsky JM, Snodgrass RG, Ono-Moore KD, Schneider DA, Newman JW, Adams SH, Hwang DH. Saturated fatty acids activate TLRmediated proinflammatory signaling pathways. J Lipid Res. 2012;53:2002-13.

67. Lupton JR, Ferrell RG. Using density rather than mass to express the concentration of gastrointestinal tract constituents. J Nutr. 1986;116:164-8.

68. Hu H, He LY, Gong Z, Li N, Lu YN, Zhai QW, Liu H, Jiang HL, Zhu WL, Wang HY. A novel class of antagonists for the FFAs receptor GPR40. Biochem Biophys Res Commun. 2009;390:557-63.

69. Houthuijzen JM, Oosterom I, Hudson BD, Hirasawa A, Daenen LGM, McLean CM, Hansen SVF, van Jaarsveld MTM, Peeper DS, Jafari Sadatmand S, et al. Fatty acid 16:4(n-3) stimulates a GPR120-induced signaling cascade in splenic macrophages to promote chemotherapy resistance. FASEB J. 2017; 31:2195-209.

70. Li R, Yu C, Li Y, Lam TW, Yiu SM, Kristiansen K, Wang J. SOAP2: an improved ultrafast tool for short read alignment. Bioinformatics. 2009;25:1966-7.

71. Zhu W, Lomsadze A, Borodovsky M: Ab initio gene identification in metagenomic sequences. Nucleic Acids Res 2010, 38:e132.

72. Li W, Godzik A. Cd-hit: a fast program for clustering and comparing large sets of protein or nucleotide sequences. Bioinformatics. 2006;22:1658-9.

73. Liu R, Hong J, Xu X, Feng Q, Zhang D, Gu Y, Shi J, Zhao S, Liu W, Wang X, et al. Gut microbiome and serum metabolome alterations in obesity and after weight-loss intervention. Nat Med. 2017;23:859-68.

74. Qin J, Li R, Raes J, Arumugam M, Burgdorf KS, Manichanh C, Nielsen T, Pons $\mathrm{N}$, Levenez F, Yamada T, et al. A human gut microbial gene catalogue established by metagenomic sequencing. Nature. 2010;464:59-65.

75. Feng Q, Liang S, Jia H, Stadlmayr A, Tang L, Lan Z, Zhang D, Xia H, Xu X, Jie $Z$, et al. Gut microbiome development along the colorectal adenomacarcinoma sequence. Nat Commun. 2015;6:6528.

76. Karlsson FH, Tremaroli V, Nookaew I, Bergstrom G, Behre CJ, Fagerberg B, Nielsen J, Backhed F. Gut metagenome in European women with normal, impaired and diabetic glucose control. Nature. 2013;498:99-103.

77. Magoc T, Salzberg SL. FLASH: fast length adjustment of short reads to improve genome assemblies. Bioinformatics. 2011;27:2957-63.

78. Edgar RC. UPARSE: highly accurate OTU sequences from microbial amplicon reads. Nat Methods. 2013;10:996-8.

79. Caporaso JG, Kuczynski J, Stombaugh J, Bittinger K, Bushman FD, Costello EK, Fierer N, Pena AG, Goodrich JK, Gordon Jl, et al. QIIME allows analysis of high-throughput community sequencing data. Nat Methods. 2010;7:335-6.

80. Qiu XY, Li X, Wu Z, Zhang F, Wang N, Wu N, Yang X, Liu YL. Fungal-bacterial interactions in mice with dextran sulfate sodium (DSS)-induced acute and chronic colitis. RSC Adv. 2016;6:65995-6006.

81. Gao X, Pujos-Guillot E, Sebedio JL. Development of a quantitative metabolomic approach to study clinical human fecal water metabolome based on trimethylsilylation derivatization and GC/MS analysis. Anal Chem. 2010;82:6447-56.

\section{Ready to submit your research? Choose BMC and benefit from:}

- fast, convenient online submission

- thorough peer review by experienced researchers in your field

- rapid publication on acceptance

- support for research data, including large and complex data types

- gold Open Access which fosters wider collaboration and increased citations

- maximum visibility for your research: over $100 \mathrm{M}$ website views per year

At BMC, research is always in progress.

Learn more biomedcentral.com/submissions 